\title{
The Cognitive Significance of Mental Files
}

\author{
Peter Pagin \\ Stockholm University \\ DOI: $10.2478 /$ disp-2013-0013 \\ BIBLID [0873-626X (2013) 36; pp. 133-145]
}

As one of the major figures in the philosophy of language and mind during a quarter of a century, François Recanati has contributed to speech act theory, the theory of meaning and truth conditions, the theory of primary pragmatic processes, to direct reference theory, and to the accounts of indirect discourse, quotation, definite descriptions, perspectival thought, and more. The topic of the new book, Mental Files, falls squarely within the philosophy of mind, but the main interest is as always semantic. Recanati is concerned with the semantics of mental representation and its relation to cognitive significance. This theme connects back to his concerns in Direct Reference. From Language to Thought, to which there are many references. Like the earlier contributions, the new book is a joy to read; clearly written, well structured, subtle in its distinctions, and forcefully argued.

In this review, I shall focus on the connection between semantics and cognitive significance, as it is developed in the book. I shall raise — what I take to be — a few problems with the account.

\section{What are mental files supposed to do?}

A central claim of Mental Files is that 'we refer through mental files, which play the role of so-called "modes of presentation" (8). Mental files are the vehicles of singular thoughts (57), and singular thoughts, in turn, are 'thoughts that are directly about individual objects, and whose content is a singular proposition - a proposition involving individual objects as well as properties' (5). 
Classically, the appeal to singular propositions as thought contents incurred a problem of cognitive significance. Romain Gary is (was) identical with Émile Ajar. He published novels under both names (used in the preceding sentence) - in fact received the Goncourt prize under each — and their coreference did not become publicly known until after his death. Pierre, who likes to read novels, entertained singular thoughts about Gary/Ajar. But he had different beliefs associated with the two names. At one point, he assented to both (1) and (2):

(1) Gary is dead.

(2) Ajar is not dead.

Apparently, Pierre has the singular belief that Gary is dead, as well as the singular belief that Ajar is not dead. Intuitively, Pierre does not believe that Ajar is dead. However, if the singular proposition that is the content of Pierre's belief that Gary is dead involves Gary himself, then it appears to be the same content as the content of a singular belief that Ajar is dead. So it seems that Pierre must believe that Ajar is dead, after all.

In order to block this conclusion, we must first recognise, with Frege, that the two names have different cognitive significance for Pierre, and then model that difference to have an effect on belief content. Achieving this is one of the tasks that Recanati assigns to mental files. ${ }^{1}$

A related task concerns empty names, or the capacity to think object-directed but objectless thoughts. An example often used in the literature is that of the astronomer Leverrier, who correctly inferred the existence of Neptune, as the cause of the perturbations of the orbit of Uranus, and incorrectly inferred that there was a planet, to be named 'Vulcan', causing the shifts of the perihelion of Mercury. No such planet exists, and so 'Vulcan' is an empty name. Still, Leverrier had Vulcan thoughts, similar in many respects to his Neptune thoughts before the actual discovery, such as

(3) Vulcan causes shifts of the perihelion of Mercury.

${ }^{1}$ See section 3 for an alternative approach to the relation between cognition and content. 
Since singular thoughts seem to require referents for their very existence, it appears impossible to account within a singular thought framework for how Leverrier could have thoughts 'about' Vulcan. But Leverrier's epistemic situation was pretty much the same as with respect to Neptune, and some singular thought theorists, including Recanati, would want to accept that Leverrier did have singular thoughts about Neptune before any direct observations of it. Explaining this is another task that Recanati sets for mental files.

For Frege, both the problem of cognitive significance and the problem of empty names was solved by means of his notion of sense, which is, or contains, a mode of presentation of an object. Frege is usually interpreted as taking the mode of presentation to be descriptive, i.e. to embody a descriptive condition, such as being the cause of the shift of the perihelion of Mercury, and this is consistent with his examples. This solves both problems. For the first, two distinct senses can present the same object, and senses are parts of thought contents. So, with 'Gary' and 'Ajar' being associated with different senses, we have an explanation, or the beginning of an explanation, of why the belief that Gary is dead can have a different content than the belief that Ajar is dead. With different contents, Pierre can believe the former but not the latter. For the second, we can accept, as Frege did, the possibility that no object at all satisfies the descriptive condition contained in the sense of e.g. 'Vulcan'. The content is well-defined and believable whether a referent exists or not.

Now, you can combine central ideas of Frege with an idea of singular thoughts by requiring that the content of a singular thought is individuated not only by the descriptive condition, but also by being about the very referent that satisfies that condition. Then no thought not about the same object could have the same content. This is the main line of the neo-Fregean idea of so-called object-dependent senses, developed by Gareth Evans 1982 and John McDowell 1977.

Recanati, however, rejects descriptivism. Mental files are to provide cognitive significance in virtue of being modes of presentation of individuals, but they are to be non-descriptive modes. In the following, we shall see how these tasks are discharged. 


\section{What are mental files?}

Mental files are the vehicles of singular thoughts. They belong to the system of mental representations. They are the mental counterparts to singular terms, and they refer, or are supposed to refer (35). More precisely, they are the counterparts to indexicals, since they possess the essential features of indexicals (57). At the same time, they are modes of presentation, which is to say 'senses' (257), and individual concepts, i.e. thought constituents (64). For someone used to thinking about language, this is puzzling. (Since it is connected to what I see as the main problems with the account, I'll pause the presentation here for a brief digression.)

We must distinguish between a linguistic expression and its semantic properties, whether it is reference or some non-extensional property, since linguistic expressions don't have their semantic properties essentially. One linguistic expression can have different meanings in different languages, different meanings at different times in the same language, and even more than one meaning in the same language at the same time. ${ }^{2}$ How can something mental both correspond to the linguistic expression and to the sense of that expression?

I have not learned to appreciate this combination. One can use the term 'concept' in accordance with the psychological tradition, to stand for mental entities rather than the contents of these entities (cf. 35, note 6), but Recanati clearly distinguishes the semantic content — the reference, or acquaintance relation — from the file itself. One might also say that what we think with are contents, and what we think with are mental representations, and hence they must be the same. But this argument may, and I think does, trade on an ambiguity of 'think with'.

In one sense we communicate 'with' sentences, and in one sense we communicate 'with' propositions, but clearly, those senses are not the same. Moreover, in some passages Recanati himself stresses the distinction between a singular thought in the sense of thought vehicle and a singular thought in the sense of thought content $(160,163)$. There, the mental counterpart of an indexical or a name — i.e. a

${ }^{2}$ Even if we make the distinction between homonymy and polysemy, the latter amounts to several (related) meanings of the same expression. 
mental file - is part of the thought vehicle only, not of the thought content. It is not easy to see how this squares with speaking of files as being senses/modes of presentation in other passages. Still, as we shall see, a file may in the end play a semantic role in virtue of its 'syntax' alone. (End of digression.)

As I have understood it, the content-involving properties of mental files are:

1) having a referent,

2) having an information channel (acquaintance relation),

3) serving a referent-intending function,

4) containing information (or misinformation) about the referent.

The most important function of a mental file is to refer to an individual, and that is exactly, according to Recanati, what almost all mental files do. Moreover, they do so in virtue of standing in a particular relation to the referent. This is the so-called indexical model, according to which the referent of a mental file is determined by means of standing in a suitable acquaintance relation to the file, not by satisfying a descriptive condition associated with it. There is a mode of presentation, but it is relational in nature, not descriptive. Recanati speaks of acquaintance relations as epistemically rewarding relations (20), or ER-relations. Acquaintance relations are epistemically rewarding in allowing the subject to gain information from the object (37). That is, there is an information channel from the object to the file, in virtue of the ER-relation.

Recanati also speaks of senses as reference-determining (118-121), but possibly this does not amount to saying more than that if two files have different referents, they also have different senses (cf. 120), in which case there is no determination-process. With certain exceptions (to which I shall return below), when a mental file exists, it stands in an acquaintance relation to an object, and so the object is ipso facto determined.

I shall discuss the third feature in Section 4. As for the fourth feature, the very term 'mental file' is connected with the idea that a mental file is a kind of repository of information, or misinformation, about the referent. This [mis-] information consists in the properties 
the subject takes the referent to have, i.e. the subject's beliefs about the referent (35). It can be thought of as a list of predicates the subject takes the referent to satisfy (37). Since predicates can be relational, and therefore involve other mental files, we cannot think of a mental file syntactically as constructed out of the predicates (on pain of a regress, if two files appear in each other's lists of predicates). Rather, the properties must be associated with the file in a non-constituting way. Information can be added to and deleted from the file, while the file itself stays the same.

There are files of different kinds. Demonstrative files are associated with a perceptual mode of presentation (74), memory files (or memory demonstratives) with a memory mode of presentation (62), and recognitional files with a recognition or familiarity mode of presentation (71). These are the varieties of basic mental files, or mental files simpliciter.

Over and above these there are non-basic, or derivative kinds. A demonstrative file is converted into a memory file when the perceptual relation to the object is lost. While the demonstrative file then goes out of existence, something remains that endures through conversions, and these are the stable files or 'piles' (82).

Some derivative files are more like proper names than like demonstratives, in that they abstract from any particular mode of presentation. These are the higher-order files, or 'encyclopedia entries', which require some acquaintance relation or other, but no particular kind (74-75).

There are also proto-files (64-65), which are distinguished from basic mental files in that they can store only information received through their own proper acquaintance relation. The proto-file SELF* of a subject stores information only through proprioception, while the proper file SELF can add information about the subject coming from other sources as well.

\section{Cognitive significance}

In the theory of meaning, we have the options of connecting semantics and cognitive significance and also of separating them. In the semantics of singular terms, Kripkean theories of rigid designation, like Kaplanian theories of direct reference, take the latter alternative. It 
is characteristic of such theories that they rely heavily on pragmatics for accounting for the content that is intuitively conveyed by means of simple sentences containing (rigid or directly referring) proper names (e.g. Soames 2002). It is also characteristic that they rely on an appeal to unexpressed senses or modes of presentation for giving the semantics of belief sentences (e.g. Salmon 1986).

Frege took the first alternative. One property, sense, both constitutes cognitive significance and serves to determine reference. It is semantic not only in the loose sense of being 'expressed', but also in the stricter sense of determining (jointly with the world) the truth values of sentences, as truth values are special cases of referents.

If we are not concerned with language, but with providing an account of mental representation, within the framework of mental files, there are still two options, but they look somewhat different. The separation alternative (corresponding to the Kripke/Kaplan alternative for public terms) cannot appeal to pragmatics, since mental files are not tools of communication, and it cannot appeal to separate modes of presentation, since the files themselves were supposed to play exactly that role. The only item that can complement the semantics is the file itself.

The connection alternative (corresponding to Fregean semantics) can be carried over to mental terms, provided the semantics of the mental terms allows it. Such an account should satisfy the following condition:

(DIF) Any difference in cognitive significance between two mental files can be explained by their semantic difference.

The analogous condition for linguistic terms is satisfied by Frege's theory. A difference of sense between two coreferring terms explains how a person can take the terms to have different referents, and by what has come to be called 'the cognitive criterion of difference' (Perry 1977), any difference in cognitive significance between two terms entails that they do have different senses.

As I have interpreted Recanati, he opts for the connection alternative. Files are supposed to serve as modes of presentation and to account for cognitive significance in virtue of that. This allows, for instance, for a difference between mental files also in the case where the subject takes the objects to be the same. According to Recanati, 
two files may be linked by a belief in the identity of the referents, and this allows information to 'flow' between the two files (what is believed of the one referent is also believed of the other; 43), but they have, or correspond to, a difference in mode of presentation nonetheless. Modes of presentation 'are supposed to account for cognitive significance, for clustering/coordination of information, and for reference determination' (8, note 3$)$. Difference in cognitive significance, then, is supposed to be reflected in difference of mental files. The fact that the subject $\mathrm{X}$ has two mental files, say alpha and beta, constitutes the fact that the modes of presentation, and hence the cognitive significance of the files are different. Since the files are different, and each file has its own information channel, the channels allow different information to 'cluster' in the different files (42, 50, 83). The referent is presented by means of the file as the object of information collected/clustered in the file.

This account will work, then, if distinct files always have distinct channels of information, or acquaintance relations. If two files can have the same channel of information, then the individuation of channels of information is insufficient to account for difference of cognitive significance. In that case, there must be something else that makes the difference.

It seems to me quite possible that a subject can have two files that do not differ in their acquaintance relations. Suppose $\mathrm{X}$ takes herself to see two moths flying around in her kitchen. She opens a file for each, alpha and beta, thinking of them as 'A' and 'B', respectively. She takes herself to see now A, now B. The acquaintance relations are indeed different in case there are two moths, one causing the opening of alpha, the other the opening of beta. But in case the subject in fact is mistaken, and there is only one moth causing the opening of both files, there does not seem to be any difference between the acquaintance relations of alpha and beta. X opens first demonstrative files, which are converted into memory files, when a moth is taken to go out of sight, and then converted to recognitional files, when a moth is taken to be seen again. There is, we may assume, no particular feature in the external aspects of the causal relations that explains the difference. $\mathrm{X}$ takes herself sometimes to see A, sometimes to see $\mathrm{B}$, and most often not to know whether she is seeing A or B. 
Such a situation is certainly possible (and so are others, essentially like it). Is there any semantic difference between the files alpha and beta? A description theorist can certainly say that the terms 'A' and 'B' differ in sense: the one can have the sense of the description 'the moth I saw first' and the other the same sense as 'the moth I saw second', even if she coined the terms only after taking herself to have seen both and did not then remember the original sightings. Maybe the term ' $\mathrm{B}$ ' then lacks a referent (and maybe not, depending on the reading of the description).

But this option is not open to the mental file theorist, since for both files there is an acquaintance relation to an object, in fact the same object, and there does not seem to be any difference between these relations except the distinctness of the mental file relata. ${ }^{3}$ Since there are two files, there is also a difference in cognitive significance, but in this case, it seems that the only difference in cognitive significance between the files, if any, is numerical distinctness of the files. ${ }^{4}$

Since distinctness of files is distinctness of mode of presentation, this is a semantic difference. So the distinctness of mental files is in itself a semantic difference. I take it that Recanati accepts this conclusion. If he does not, he faces the challenge of explaining away the possibility. But I also take it that he holds that a mere difference in syntax cannot in itself constitute a semantic difference (linguistic

\footnotetext{
${ }^{3}$ Could one nonetheless find such a difference? Krista Lawlor has pressed this question. From the description of the case, I have no definitive proof that one cannot, but I also see no way of doing it. Suppose we have a sub-type of the scenario where one perceptual gestalt triggers alpha while another gestalt triggers beta (and yet others are neutral). The subject incorrectly takes these two gestalts to correspond to observational properties not shared between A and B. Would such a difference between alpha and beta be a difference between their acquaintance relations? As far as I can see, the gestalts would be pieces of information within the files rather than ingredients in the relations. For instance, the subject could change her belief about the observational properties of the object without changing the identity of the file.

${ }^{4}$ As Kripke (1980: 81) pointed out, a person can associate the same sense of e.g. two historical names, like the names of two physicists, Feynman and GellMann, taking them to have different referents, while yet not knowing anything about the one that she does not also know about the other. But in that case, unlike the present mental files case, the senses are incomplete (or at least taken to be incomplete): not sufficient to determine unique bearers.
} 
quotation contexts aside).

The result seems to be that the (DIF) condition isn't met, and that Recanati ends up with the separation alternative rather than with the connection alternative, a result I think he does not want to embrace. The way out I guess Recanati would choose here, is to appeal to his idea of primary content (24), distinct from the referential, secondary content. That the file itself can make a semantic difference over and above reference, and over and above the acquaintance relation, may be acceptable in light of this further level of content, at least if two files must differ in primary content.

Like Frege's notion of sense in part was meant to account for the possibility of thoughts without referents, so the notion of primary content of mental files is meant to account for the possibility of singular thought without acquaintance. We now turn to that.

\section{Required acquaintance and primary content}

On 155 Recanati sums up the mental files framework in two principles:

1. The subject cannot entertain a singular thought about an object $a$ without possessing, and exercising, a mental file whose referent is $a$.

2. To possess and exercise a mental file whose referent is $a$ the subject must stand in some acquaintance relation to $a$.

Surprisingly, Recanati goes on to deny that it follows that "no singular thought can be entertained unless the subject is acquainted with what her thought is about'. This is achieved by interpreting the second principle as a normative claim. That is, as I understand it, Principle 2 does not state that acquaintance is a necessary condition on mental files, only that it is a necessary condition on mental files that they are required to have an acquaintance relation to an object. Since a file can be required to have such a relation without actually having it, the conclusion is blocked, as desired.

The possibility of singular thought without acquaintance depends on the appeal to its function: 
'But singular thought involves tokening a singular vehicle in thought (a mental file, or a mental name); and a singular vehicle, qua type, is individuated in terms of its function, which is: the storing of information gained through acquaintance. So singular thought is still defined in terms of acquaintance, even if there can be singular thought in the absence of (present or even future) acquaintance.' (163-63, emphasis in the original.)

It is also clear, I think, that the function referred to here is what is in other passages called the primary content:

'The primary content of a thought constituent is taken to be its function or role, which function or role contextually determines its referential or 'secondary' content' (24).

The primary content, then, allows thinking singular thoughts without standing in an acquaintance relation to the object. 'Singular thought' here means singular thought vehicle (164): without the acquaintance relation no singular content is established. ${ }^{5}$

I find the idea of primary content problematic. The concept of a mental file, like the concept of a gene in genetics, is in itself functional. Unlike linguistic expressions, we don't observe mental files or tokenings of them. We cannot demonstrate them. What we know about mental files, we know on the basis of the theory of mental files. 'Mental file' is a theoretical term in a theory like Recanati's, and the interpreted theory formulation it occurs in gives us its functional role, which is our concept of a mental file. We can then go on to ask whether there exist mental entities that fill this role, and whether there is a unique collection of entities that do. This is just the customary predicament of theoretical terms in science. Certain observations — in the present case, for instance, certain introspective observations - may confirm or disconfirm the theory through its observational consequence. So far, all is in order.

The problematic step comes when an additional functional role or normative requirement is included in the basic functional role itself. This is not to say that a normative requirement cannot be included

${ }^{5}$ Still, Recanati oscillates between this position and a more 'instrumental' position where one does have a singular thought about an object if one opens a file for it, even without acquaintance, provided the circumstances are optimal, like in the case of Leverrier and Neptune, when the subject is justified in expecting a future acquaintance (Cf. 167, note 9). 
with a function. To take an example, the president of the United States is required to ask Congress to approve his budget. The presidency is an office, i.e. a functional role, fulfilled by one person at a time. It is part of serving in that office that one is required to ask Congress for approval. This is fine, but it is fine because there is an independent method of identifying the holder of the office. The holder is identified through a national election and the ceremony of being sworn into office. Suppose, by contrast, that there were no such independent method of identifying the president, and that it were wholly a matter of finding out who fulfills the functional role. In trying to find this out, we would then ask, for instance, who the generals take their orders from, but as part of the quest of finding the president, we would also ask persons whether they are required to ask Congress to approve their budget. But this does not make sense, since one can be subject to such a requirement only as a consequence of serving in the office. The person asked if she is so required can only answer 'I don't know. Yes, if I am the president, and No, if I am not'. The president must be identified independently of being subject to the requirements of the office.

In classical functional role theory (e.g. Loar 1986), the functional roles are causal. This means that we can investigate the causal pattern in e.g. processes of the brain, to find out what brain states fulfill certain functional roles. But when the functional role is normative, this cannot be done. We cannot, as a means of identifying mental files, get hold of a brain state, or a mental state, and ask whether that state is required to have an acquaintance relation to an object. A brain state, or a mental state, can at most be subject to such a requirement once it has been identified as a mental file by an independent criterion. For instance, if mental files were all causally related to external objects by some acquaintance relation, we could use that general fact to identify mental files. But this is not the case, on Recanati's account. It is only part of their function to be required to stand in an acquaintance relation. But that means conflating a consequence of being a mental file with what is needed for identifying them in the first place. That is why I find the idea of the primary content of mental files problematic.

If the idea of primary content is removed from the theory, I guess the idea of singular thought without acquaintance will go by the 
board. But then the mental file as a part of a singular theory vehicle will be reduced to mere syntax in the system of mental representation. And mere syntax does not account for cognitive significance, at least not on the connection account. So if, as was argued in the preceding section, there are differences in cognitive significance that go beyond differences in acquaintance relations, the mental files framework does not provide a complete account of cognitive significance either. $^{6}$

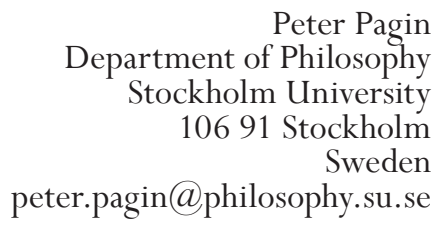

\section{References}

Evans, Gareth. 1982. The Varieties of Reference, ed. by John McDowell. Oxford: Clarendon Press.

Kripke, Saul. 1980. Naming and Necessity. Cambridge, Mass.: Harvard University Press.

Loar, Brian. 1986. Mind and Meaning. Cambridge: California University Press.

McDowell, John. 1977. On the Sense and Reference of a Proper Name. Mind 86: 159-85.

Perry, John. 1977. Frege on Demonstratives. The Philosophical Review 86: 474-97.

Recanati, François. 1993. Direct Reference. From Language to Thought. Oxford: Blackwell.

Recanati, François. 2013. Mental Files. Oxford: Oxford University Press.

Salmon, Nathan. 1986. Frege’s Puzzle. Cambridge, Mass.: MIT Press.

Soames, Scott. 2002. Beyond Rigidity: the Unfinished Semantic Agenda of Naming and Necessity. Oxford: Oxford University Press.

${ }^{6}$ Thanks for helpful comments to Kathrin Glüer and Krista Lawlor. 